\title{
Dynamical Systems on Three Manifolds Part I: Knots, Links and Chaos
}

\author{
Yi SONG, Stephen P. BANKS and David DIAZ \\ Department of Automatic Control and Systems Engineering, \\ University of Sheffield, Mappin Street, \\ Sheffield, S1 3JD. \\ e-mail: s.banks@sheffield.ac.uk
}

November 1, 2018

\begin{abstract}
In this paper, we give an explicit construction of dynamical systems (defined within a solid torus) containing any knot (or link) and arbitrarily knotted chaos. The first is achieved by expressing the knots in terms of braids, defining a system containing the braids and extending periodically to obtain a system naturally defined on a torus and which contains the given knotted trajectories. To get explicit differential equations for dynamical systems containing the braids, we will use a certain function to define a tube neigbourhood of the braid. The second one, generating chaotic systems, is realized by modelling the Smale horseshoe.
\end{abstract}

Keywords: knots, braids, chaotic systems, Smale horseshoe, $C^{\infty}$ functions.

\section{Introduction}

Knot theory has been an important subject in its own right for a long time (see [Kauffman, 1987]), and recently a great deal has been written on the connections between knot theory and dynamical systems [Ghrist et al, 1997]. The key idea is this: a closed (periodic) orbit in a three-dimensional flow is an embedding of the circle, $S^{1}$, into the three-manifold that constitutes the state space of the system, hence it is a knot (see [Kauffman, 1987]). Hence, periodic solutions of dynamical systems may be knotted or linked, and, in fact, a chaotic system contains any knot and link ([Birman and Williams, 1983]). A simple approach to obtaining a (non-chaotic) system which contains an arbitrary knot (even a wild knot) is given in [Banks and Diaz, 2004].

It is widely known that any knot can be expressed in terms of braids, so in the first part of this paper we propose to show how to write down general 
explicit differential equations for these braids over a finite time interval, and then by making the vector field periodic, we can glue the two ends of the phase space at successive periodic time points together (see Fig 1), which will give us the desired knot embedded within a solid torus.

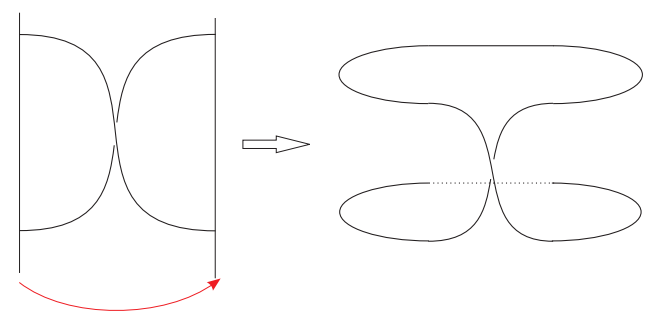

Figure 1: Constructing knots from braids by gluing the two ends together

This is achieved by using $C^{\infty}$ functions to make the twists in the braids. We shall find that any braid in a solid torus is given by an equation of the form

$$
\left\{\begin{array}{l}
\dot{x}=\sum_{i} f_{i}(x, y, z) \\
\dot{y}=\sum_{i} g_{i}(x, y, z) \\
\dot{z}=c
\end{array}\right.
$$

where $c$ is a constant and $f$ and $g$ are $C^{\infty}$ functions (i.e. an infinitely differentiable functions) which are identically 1 on an interval $(-\infty, a)$ and identically 0 on an interval $(b, \infty)$, with $a<b$. From the equations above, our dynamical system is defined by the sum of a sequence of $C^{\infty}$ functions (combined in a proper way). However, different strands of the braids will certainly affect each other, which makes it difficult to control the global behaviour of the whole system, especially at the two ends, where we want the vector field to fit together smoothly. This again involves the introduction of a $C^{\infty}$ function to control the whole dynamical system so that the vector fields corresponding to different strands in our braid will not interact.

We shall introduce the algorithm to express knots in terms of braids in the next section. This result is certainly well known, but we include this for the convenience of the reader and to fix the notation and ideas for the rest of the work. Then in $\S 3$ we outline the main idea of $C^{\infty}$ functions that we need to generate dynamical systems for braids, which will glue together and be knots, as will be stated in $\S 4$. Finally, we will consider how to use similar ideas to create chaotic systems (containing no homoclinic orbits) in $\S 5$.

In the second part of the paper we shall consider a more general approach to the study of dynamical systems on three manifolds, using Heegaard splittings and the theory developed recently in [Banks \& Song, 2006] for the structure of general dynamical systems on surfaces, using automorphic function theory. 


\section{Relation between Knots and Braids}

We shall generate systems containing any given knot by expressing the knot in the form of a closed braid. Then we determine a system with a periodic vector field which contains the desired braid. First we outline Alexander's theorem relating braids to knots.

A knot is a smooth embedding of the circle $S^{1}$ in $\mathbb{R}^{3}$ (see Fig 2), and a link is a finite disjoint collection of knots. In this paper we only consider knots, but the ideas generalize easily to links.

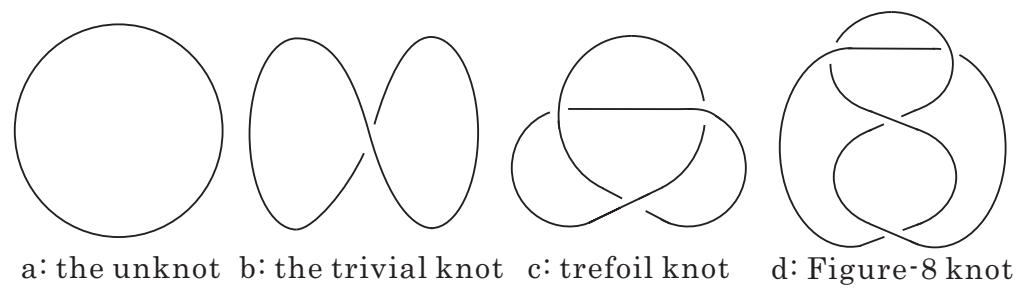

Figure 2: The simplest knots

Knot theory is studied conveniently in terms of braids, which were originally introduced by [Artin, 1925]. By definition, an m-strand braid is a set of $m$ non-intersecting smooth paths connecting $m$ points on one horizontal line to $m$ points on another horizontal line (below the first one) - see [Kauffman,1987] and Fig 3. If we glue the corresponding left and right hand side of the braid together respectively, we get the so-called closure of a braid. From Fig 3, we see that it is actually a knot. More generally, the closure of a braid is a link. Usually closures of braids are taken to be oriented, all strands of the braid are oriented from left to right in this paper.

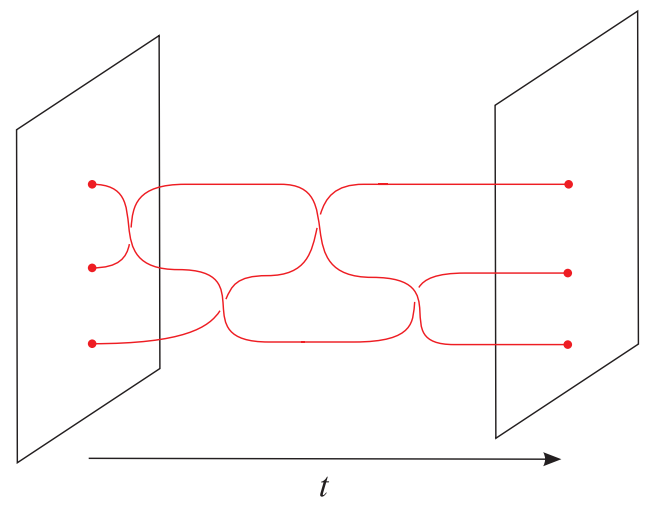

Figure 3: A braid in 3-space 
Theorem 2.1 (Alexander's theorem) Each link can be represented as the closure of a braid.

Since a link is a smooth embedding of several disjoint circles in $\mathbb{R}^{3}$, it is actually composed of several knots. Consequently, we have the following corollary.

Corollary 2.1 Each knot can be represented as the closure of a braid.

Now we give an algorithm to construct braid from a given knot. We will illustrate the method by the use of a figure- 8 knot - the general case will then be clear. (see Fig 4 ).

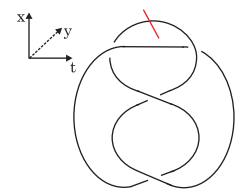

a

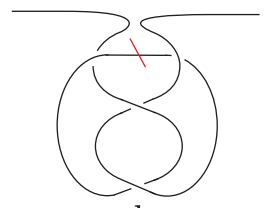

$\mathrm{b}$

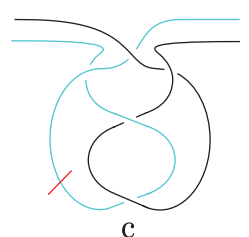

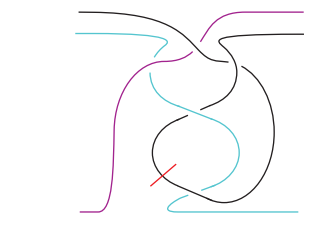

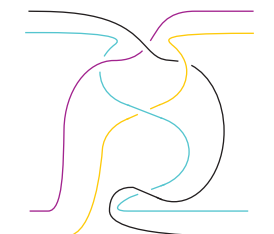

e

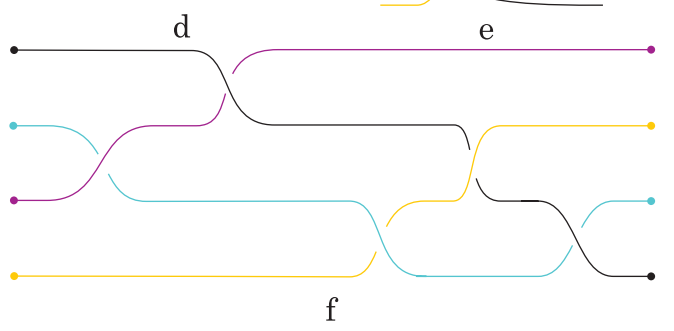

Figure 4: Construction of a braid from the figure- 8 knot

Since a knot is essentially a closed loop, while the braid construction from a knot has at lease one strand (in the unknot case), and each strand has two ends, basically what we should do is to cut the knot in a proper way so that it will turn into several strands which give us the correct braid representation.

First, we choose one part of the given knot which is away from the crossings, cut the diagram at a point from this part and straighten the two ends resulted from the cut, which gives us the first strand of the braid. Obviously it is better to cut the top or the bottom of the knot, as shown in Fig 4 (a) and (b). 
As we know, the only knot that has just a one-string braid is the unknot, which implies that all the other knots have more than one strand in the corresponding braid construction. This means in order to build up the braid representation, there is a need to cut the diagram again to get the remaining strands. Self-crossings of any strand of the braid can be removed by Reidemeister moves. So the next step will be choosing a part of the resulting diagram which is before or after a self-crossing of the one-strand from the first step, cut it and straighten the diagram as we did previously. A little care must be taken at this place, since a knot is just one circle and so for each strand in the braid representation, we must guarantee that it starts at one end and finishes at the other. The solution to this is simple: we just need to add another crossing if necessary to ensure that a strand comes in from one side must go out from the other side. (see Fig 4 (c) and (d) for an illustration) Meanwhile, for each strand, the starting and ending points cannot share the same $x$-value or $y$-value (according to the coordinate as shown in Fig 4), otherwise the braid represents a multi-component link instead of a knot.

Perform this operation repeatedly until all the strands in the braid have no self-crossings, as shown in Fig 4 (e).

Finally, rearrange the braid so that there is at most one twist at each vertical strip (as shown in Fig $4(\mathrm{f})$ )).

Eventually we get a braid representation of the knot. Of course, there are an infinite number of braid representations of a given knot; however, we will choose the simplest one to study in this paper.

\section{$3 \quad C^{\infty}$ Functions}

Now we shall give a brief resume of the theory of $C^{\infty}$ functions which we need in the next section. All the results are well known, and can be found, for example in [Helgason, 1978].

Let $\mathbb{R}^{m}$ and $\mathbb{R}^{n}$ denote two Euclidean spaces of $m$ and $n$ dimensions, respectively. Let $S$ and $S^{\prime}$ be open subsets of $\mathbb{R}^{m}$ and $\mathbb{R}^{n}$, respectively, and suppose $\psi$ is a mapping from $S$ to $S^{\prime}$.

Definition 3.1 The mapping $\psi$ is called differentiable if the coordinates $y_{j}(\psi(p))$ of $\psi(p)$ are differentiable functions of the coordinates $x_{i}(p), p \in S$.

Definition 3.2 The mapping $\psi$ is called analytic if for each point $p \in S$ there exists a neighbourhood $U$ of $p$ and $n$ power series $P_{j}(1 \leq j \leq n)$ in $m$ variables such that $y_{j}(\psi(q))=P_{j}\left(x_{1}(q)-x_{1}(p), \cdots, x_{m}(q)-x_{m}(p)\right)$ $(1 \leq j \leq n)$ for $q \in U$.

Definition 3.3 A differentiable mapping $\psi: O \rightarrow O^{\prime}$ is called a diffeomorphism of $O$ and $O^{\prime}$ if, $\psi$ is one-to-one and onto, and the inverse mapping $\psi^{-1}$ is differentiable. 
For an analytic function on $\mathbb{R}^{m}$, if it vanishes on an open set, then it is identically zero. However, for general differentiable functions and in particular $C^{\infty}$ functions, the situation is completely different.

Definition 3.4 If $A$ and $B$ are two disjoint subsets of $\mathbb{R}^{m}$, then there exists an infinitely differentiable function $\varphi$ which is identically 1 on $A$ and identically 0 on $B$. To emphasize the dependence on $A$ and $B$ we often write this as $\varphi(x ; A, B)$.

Obviously such a function is non-analytic, since it is identically 0 or 1 for a continuous interval; but it is infinitely differentiable, which makes it very useful in the next section.

The standard procedure for constructing such a $C^{\infty}$ function is as follows: Let $0<a<b$ and consider the function $f$ on $\mathbb{R}$ defined by

$$
f(x)= \begin{cases}\exp \left(\frac{1}{x-b}-\frac{1}{x-a}\right) & \text { if } a<x<b \\ 0 & \text { otherwise }\end{cases}
$$

Then $f$ is differentiable and the same holds for the function

$$
F(x)=\frac{\int_{x}^{b} f(t) d t}{\int_{a}^{b} f(t) d t},
$$

which has value 1 for $x \leq a$ and 0 for $x \geq b$. The $C^{\infty}$ function $\varphi$ defined on $\mathbb{R}^{m}$ is

$$
\varphi\left(x_{1}, \cdots, x_{m}\right)=F\left(x_{1}^{2}+\cdots+x_{m}^{2}\right) .
$$

It can be seen that $\varphi$ is differentiable and has values 1 for $x_{1}^{2}+\cdots+x_{m}^{2} \leq a$ and 0 for $x_{1}^{2}+\cdots+x_{m}^{2} \geq b$, by a slight abuse of notation we shall write it as $\varphi(x ; a, b)$. (see Fig 5 )

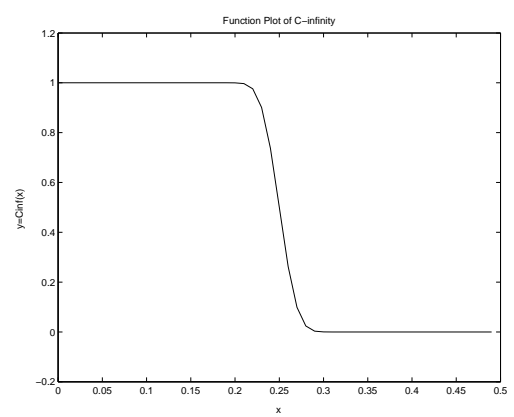

Figure 5: Function plot of $C^{\infty}$

In fact we can approximate it by just using an exponential function, say $y=\exp \left(-x^{20}\right)$. However, for exact matching at the boundaries we require a function which is constant on certain regions of space. 


\section{Dynamical Systems for Braids}

We now consider dynamical systems which contain any braids constructed from some given knots. Using the coordinate system shown in Fig 4, we notice that a braid is composed of several strands and twists, with at most one twist at a certain vertical strip (an interval of the $t$ coordinate). Hence if we can find a dynamical system which gives us the twist, then it just remains to repeat the process to give the appropriate number of twists for the complete braid.

Each strand in the braid is defined by a set of equations of the form

$$
\begin{aligned}
\dot{x} & =G_{1}(x, y, t) \\
\dot{y} & =G_{2}(x, y, t) \\
\dot{z} & =G_{3}(x, y, t)
\end{aligned}
$$

where $G_{1}, G_{2}$ and $G_{3}$ are some functions of $x, y$ and $t$. Normally we take $G_{3}$ to be a constant so the $z$-axis is effectively the time axis and periodicity of $G$ with respect to $t$ will lead to a system with the required knot.
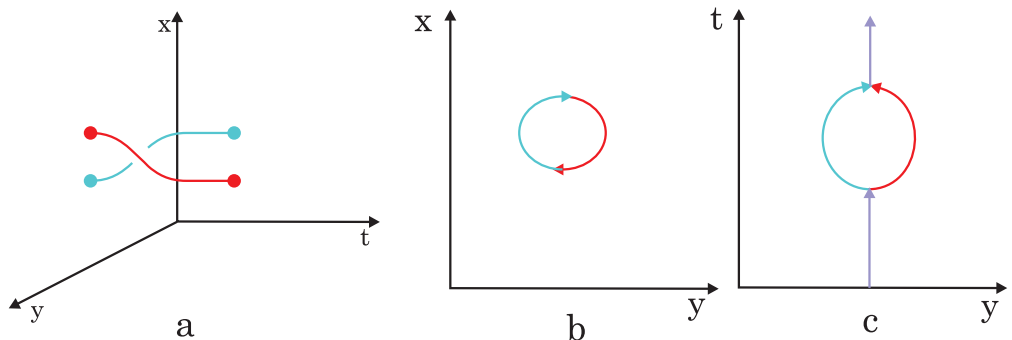

Figure 6: One twist of a braid

Fig 6] shows a twist projected onto three different planes, namely the $x z-, x y-$, and $z y$-planes, respectively.Because of the need to glue the braid together to get a knot, we assume that at the two ends, all the strands are parallel to the $z$-axis, (mathematically speaking, we need $\dot{x}=0, \dot{y}=0$ ) which makes the vector fields at these connecting points match at the periodic points. We now give explicit equations for these strings.

As shown in Fig 6, in the $x z$-plane projection, the shape of the red strand is that of the $C^{\infty}$ function. After studying the change of the vector field, $\dot{x}$, with respect to $t$, we get

$$
\dot{x}=\varphi(t ; a, b)-\varphi(t ; b, c), \quad(a<b<c) .
$$

In case of an ascending strand instead of the descending one, we have

$$
\dot{x}=\varphi(t ; b, c)-\varphi(t ; a, b), \quad(a<b<c) .
$$


In the $x y$-plane, the transformation group acts as a circle, which brings the top one to the bottom and bottom to the top without intersection. In the $z y$-plane, the trajectory is a semicircle in the middle plus two straight lines at the two ends. A proper combination of $C^{\infty}$ functions will give any desired link. Thus, for an over-crossing, such as the red one in Fig 6, we have

$$
\dot{y}=\varphi(t ; b, c)-\varphi(t ; a, b)+\varphi(t ; c, d)-\varphi(t ; d, e) \quad(a<b<c<d<e)
$$

while for an under-crossing, such as the blue one, it becomes

$$
\dot{y}=\varphi(t ; a, b)-\varphi(t ; b, c)+\varphi(t ; d, e)-\varphi(t ; c, d) \quad(a<b<c<d<e)
$$

We assume that $\dot{z}$ is a constant and set $z=t$; then the equation for one twist in a braid is

$$
\begin{aligned}
& \dot{x}=\left\{\begin{array}{l}
\varphi(t ; a, b)-\varphi(t ; b, c) \\
\varphi(t ; b, c)-\varphi(t ; a, b)
\end{array} \quad(a<b<c)\right. \\
& \dot{y}=\left\{\begin{array}{l}
\varphi(t ; b, c)-\varphi(t ; a, b)+\varphi(t ; c, d)-\varphi(t ; d, e) \\
\varphi(t ; a, b)-\varphi(t ; b, c)+\varphi(t ; d, e)-\varphi(t ; c, d)
\end{array}\right. \\
& (a<b<c<d<e) \\
& \dot{z}=\text { constant }
\end{aligned}
$$

where the choice is made depending on whether the twisted strand is ascending or descending, under-crossing or over-crossing.

Then we can get the dynamical system for just one strand, it is of the form

$$
\begin{aligned}
& \dot{x}=\sum_{i=1}^{p} \pm\left(\varphi\left(t ; a_{i}, b_{i}\right)-\varphi\left(t ; b_{i}, c_{i}\right)\right) \quad\left(a_{i}<b_{i}<c_{i}\right) \\
& \dot{y}=\sum_{i=1}^{p} \pm\left(\varphi\left(t ; a_{i}, b_{i}\right)-\varphi\left(t ; b_{i}, c_{i}\right)+\varphi\left(t ; d_{i}, e_{i}\right)-\varphi\left(t ; c_{i}, d_{i}\right)\right) \\
& \left(a_{i}<b_{i}<c_{i}<d_{i}<e_{i}\right) \\
& \dot{z}=\text { constant }
\end{aligned}
$$

where $p$ is total number of twists in this strand, the \pm sign is taken depending one whether at the corresponding twist $i$, the strand ascends or descends, under-crosses or over-crosses.

The next step will be to combine all the equations for different strands together in a proper way so that we get a final one for the whole braid. As shown below in Fig 7, we create a tube around each string, such that within this tube, all the trajectories follow the middle strand, while outside it, the dynamics are all zero, which means both $\dot{x}$ and $\dot{y}$ are 0 . 
By definition, an $m$-strand braid is a set of $m$ non-intersecting smooth paths, so the key idea is this: as long as the radius is small enough, there exists a tube around each string that has no intersection with others. In this way, we effectively get one equation for the whole braid while avoiding the interaction between the stands.

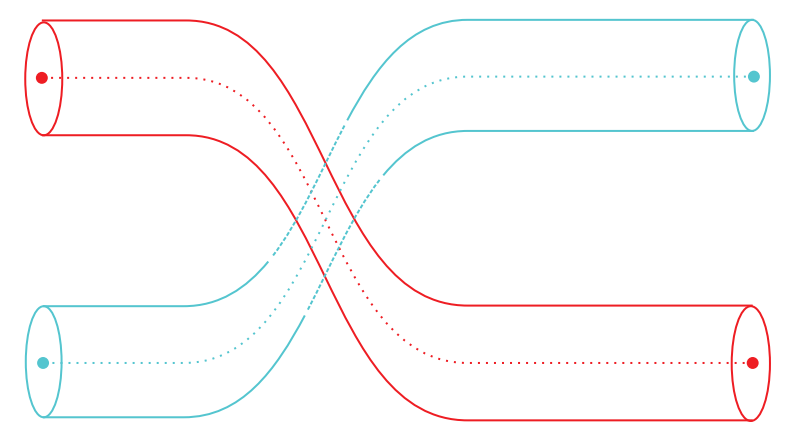

Figure 7: Creating a tube around each existing strand

This is also achieved by using the $C^{\infty}$ function of the form

$$
\left.\varphi=\varphi\left(\left(x-x_{1}\right)^{2}+\left(y-y_{1}\right)^{2}\right) ; a, b\right)
$$

where $\left(x_{1}, y_{1}\right)$ is the coordinate of the middle strand with respect to different $t$ value, and $a, b$ need to be chosen carefully so that they are small enough to avoid intersection with other tubes.

Consequently, the dynamical system of the braid is

$$
\begin{aligned}
\dot{x} & =\sum_{j=1}^{q} \varphi_{j} \cdot \dot{x}_{j} \\
\dot{y} & =\sum_{j=1}^{q} \varphi_{j} \cdot \dot{y}_{j} \\
\dot{z} & =\text { constant }
\end{aligned}
$$

where $q$ is the total number of strands in the braid, $\varphi_{j}$ is the tube function for the $j$ th strand, and $\dot{x}_{j}, \dot{y}_{j}$ are the dynamics for the $j$ th strand obtained from Equation (10). As before we choose $\dot{z}$ to be a constant, so $z$ is like a time-axis.

Example 4.1 Consider the trefoil knot shown in Fig 2, we shall give two braid presentations and the corresponding dynamical systems for it.

i) As shown in Fig 8 , the trefoil knot can be represented by a 2 -strand braid. Hence the dynamics for strand 1 is of the form 


$$
\begin{aligned}
\dot{x}= & \varphi(t ; a, b)-\varphi(t ; b, c)+\varphi(t ; e, f)-\varphi(t ; d, e)+\varphi(t ; g, h)-\varphi(t ; h, i) \\
\dot{y}= & \varphi\left(t ; a, \frac{a+b}{2}\right)-\varphi\left(t ; \frac{a+b}{2}, b\right)+\varphi\left(t ; \frac{b+c}{2}, c\right)-\varphi\left(t ; b, \frac{b+c}{2}\right) \\
& +\varphi\left(t ; \frac{d+e}{2}, e\right)-\varphi\left(t ; d, \frac{d+e}{2}\right)+\varphi\left(t ; \frac{e+f}{2}, f\right)-\varphi\left(t ; e, \frac{e+f}{2}\right) \\
& +\varphi\left(t ; g, \frac{g+h}{2}\right)-\varphi\left(t ; \frac{g+h}{2}, h\right)+\varphi\left(t ; \frac{h+i}{2}, i\right)-\varphi\left(t ; h, \frac{h+i}{2}\right) \\
\dot{z}= & \text { constant }
\end{aligned}
$$

for some numbers $a, b, c, d, e, f, g, h, i$, while for strand 2 , the equations are much the same except the change of plus/minus sign. So let $\left(x_{1}, y_{1}\right)$ and $\left(x_{2}, y_{2}\right)$ stand for the $x$ - and $y$-value for strand 1 and 2 respectively, we can build up a tube around each string, and finally get the dynamical system for the braid, which is

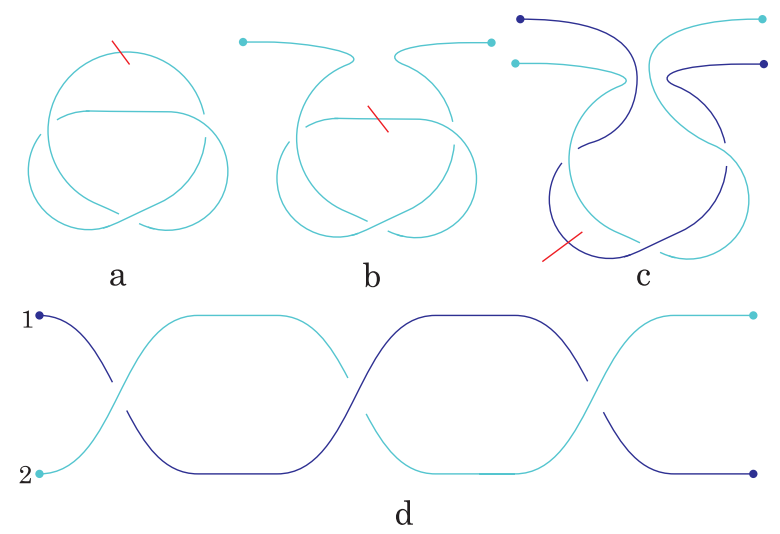

Figure 8: Braid construction of the trefoil knot - method 1

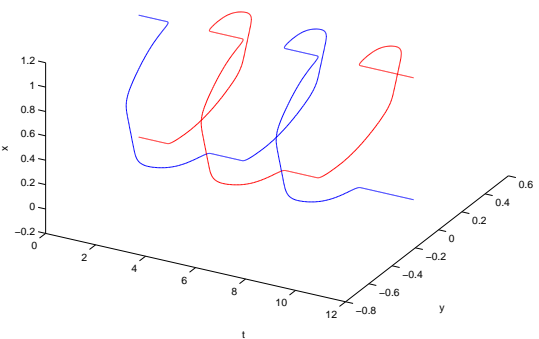

Figure 9: A 2-strand braid generated from Matlab 


$$
\begin{aligned}
\dot{x}= & \left(\left(x-x_{1}\right)^{2}+\left(y-y_{1}\right)^{2} ; \xi_{1}, \xi_{2}\right) \times \\
& (\varphi(t ; a, b)-\varphi(t ; b, c)+\varphi(t ; e, f)-\varphi(t ; d, e)+\varphi(t ; g, h)-\varphi(t ; h, i)) \\
& +\varphi\left(\left(x-x_{2}\right)^{2}+\left(y-y_{2}\right)^{2} ; \xi_{1}, \xi_{2}\right) \times \\
& (\varphi(t ; b, c)-\varphi(t ; a, b)+\varphi(t ; d, e)-\varphi(t ; e, f)+\varphi(t ; h, i)-\varphi(t ; g, h)) \\
\dot{y}= & \varphi\left(\left(x-x_{1}\right)^{2}+\left(y-y_{1}\right)^{2} ; \xi_{1}, \xi_{2}\right) \times \\
& \left(\varphi\left(t ; a, \frac{a+b}{2}\right)-\varphi\left(t ; \frac{a+b}{2}, b\right)+\varphi\left(t ; \frac{b+c}{2}, c\right)-\varphi\left(t ; b, \frac{b+c}{2}\right)\right. \\
& +\varphi\left(t ; \frac{d+e}{2}, e\right)-\varphi\left(t ; d, \frac{d+e}{2}\right)+\varphi\left(t ; \frac{e+f}{2}, f\right)-\varphi\left(t ; e, \frac{e+f}{2}\right) \\
& \left.+\varphi\left(t ; g, \frac{g+h}{2}\right)-\varphi\left(t ; \frac{g+h}{2}, h\right)+\varphi\left(t ; \frac{h+i}{2}, i\right)-\varphi\left(t ; h, \frac{h+i}{2}\right)\right) \\
& +\varphi\left(\left(x-x_{2}\right)^{2}+\left(y-y_{2}\right)^{2} ; \xi_{1}, \xi_{2}\right) \times \\
& \left(\varphi\left(t ; \frac{a+b}{2}, b\right)-\varphi\left(t ; a, \frac{a+b}{2}\right)+\varphi\left(t ; b, \frac{b+c}{2}\right)-\varphi\left(t ; \frac{b+c}{2}, c\right)\right. \\
& +\varphi\left(t ; d, \frac{d+e}{2}\right)-\varphi\left(t ; \frac{d+e}{2}, e\right)+\varphi\left(t ; e, \frac{e+f}{2}\right)-\varphi\left(t ; \frac{e+f}{2}, f\right) \\
& \left.+\varphi\left(t ; \frac{g+h}{2}, h\right)-\varphi\left(t ; g, \frac{g+h}{2}\right)+\varphi\left(t ; h, \frac{h+i}{2}\right)-\varphi\left(t ; \frac{h+i}{2}, i\right)\right) \\
\dot{z}= & \operatorname{constant}
\end{aligned}
$$

where $\xi_{1}, \xi_{2}$ have to be chosen carefully to avoid intersection of different tubes.

Using Matlab, we get a plot of this 2-strand braid, as shown in Fig 9 ,

ii) By adding another cut as shown in Fig 8 (c), we have a new braid presentation for the same trefoil knot, as in Fig 10.

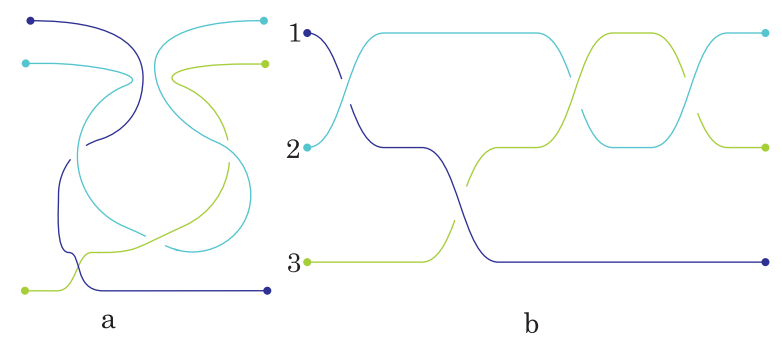

Figure 10: Braid construction of the trefoil knot - method 2

In the same manner as in method 1 , let $\left(\dot{x}_{1}, \dot{y}_{1}, \dot{z}_{1}\right),\left(\dot{x}_{2}, \dot{y}_{2}, \dot{z}_{2}\right),\left(\dot{x}_{3}, \dot{y}_{3}, \dot{z}_{3}\right)$ stand for the dynamics, $\left(x_{1}, y_{1}\right),\left(x_{2}, y_{2}\right)$ and $\left(x_{3}, y_{3}\right)$ for the $x$ - and $y$-value of the three strings, respectively. Then the dynamical system for this 3 -strand braid is 


$$
\begin{aligned}
\dot{x}= & \varphi\left(\left(x-x_{1}\right)^{2}+\left(y-y_{1}\right)^{2} ; \xi_{1}, \xi_{2}\right) \times \dot{x}_{1} \\
& +\varphi\left(\left(x-x_{2}\right)^{2}+\left(y-y_{2}\right)^{2} ; \xi_{1}, \xi_{2}\right) \times \dot{x}_{2} \\
& +\varphi\left(\left(x-x_{3}\right)^{2}+\left(y-y_{3}\right)^{2} ; \xi_{1}, \xi_{2}\right) \times \dot{x}_{3} \\
\dot{y}= & \varphi\left(\left(x-x_{1}\right)^{2}+\left(y-y_{1}\right)^{2} ; \xi_{1}, \xi_{2}\right) \times \dot{y}_{1} \\
& +\varphi\left(\left(x-x_{2}\right)^{2}+\left(y-y_{2}\right)^{2} ; \xi_{1}, \xi_{2}\right) \times \dot{y}_{2} \\
& +\varphi\left(\left(x-x_{3}\right)^{2}+\left(y-y_{3}\right)^{2} ; \xi_{1}, \xi_{2}\right) \times \dot{y}_{3} \\
\dot{z}= & \text { constant }
\end{aligned}
$$

A picture for this 3-strand braid generated from Matlab is shown in Fig 11.
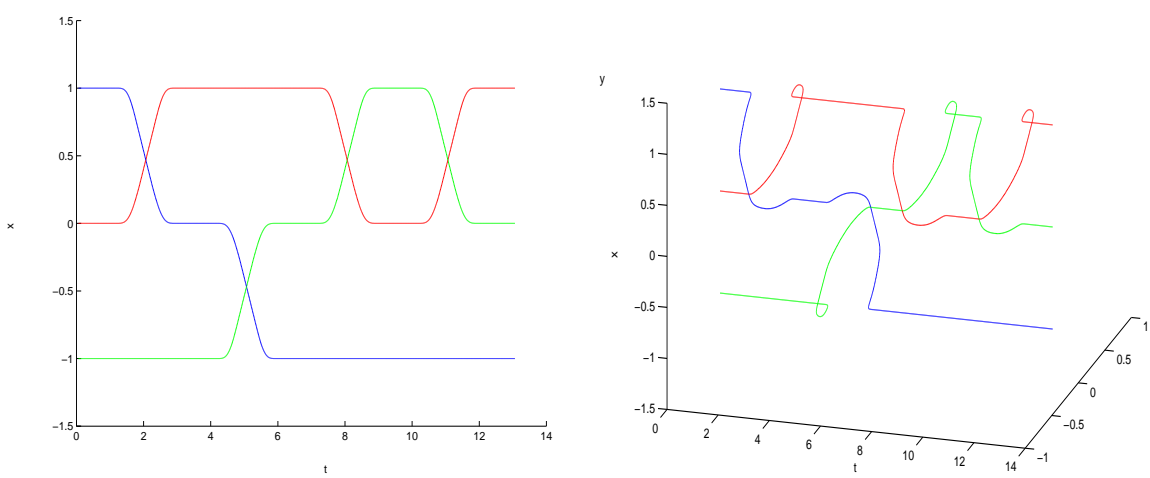

Figure 11: A three strand braid presentation for the trefoil knot

After gluing the corresponding ends of the braid together, we get the required knot situated in a solid torus - this is equivalent to making the vector fields in the systems above periodic.

\section{Chaotic Systems}

In this section we shall demonstrate how to obtain dynamical systems with arbitrarily knotted chaos. We shall do this by making an extension of the methods in the previous sections. In particular, we shall need some elementary ideas from transformation group theory. Thus, if $X$ is a topological space, and $G$ is a group, we say that $G$ is a transformation group on $X$ if there is a continuous map $\varphi: G \times X \rightarrow X$ such that

(i) $\varphi(g, \varphi(h, x))=\varphi(g h, x)$ for all $g, h \in G$, and all $x \in X$

(ii) $\varphi(e, x)=x$ for all $x \in X$, where $e$ is the identity of $G$.

We usually write $g x$ for $\varphi(g, x)$. If $G$ is a subset of $G l(n)$ (the general linear group), we call $G$ a linear transformation group. 
Consider now a process for modifying a given dynamical system

$$
\dot{x}=f(x, t)
$$

by a given function $t \rightarrow G(t)$ where $G(t)$ is an element of a (linear) transformation group for each $t$. We define

$$
y(t)=G(t) x(t) .
$$

Then

$$
\begin{aligned}
\dot{y} & =\dot{G} x+G \dot{x} \\
& =\dot{G} G^{-1} y+G f(x, t) \\
& =\dot{G} G^{-1} y+G f\left(G^{-1} y, t\right) \triangleq \tilde{G}(y, t)
\end{aligned}
$$

Theorem 5.1 Suppose that the vector field $(x, t) \rightarrow f(x, t)$ (defined on a subset $U \subseteq \mathbb{R}^{n}$ ) is periodic in $t$, with period $\pi$, and that the map $t \rightarrow G(t)$, where $G(t)$ belongs to some linear transformation group on $U$, is such that the vector field

$$
(y, t) \rightarrow \dot{G}(t) G^{-1}(t) y+G(t) f\left(G^{-1}(t) y, t\right)
$$

is also periodic in $t$ with period $\pi$, then the system

$$
\left\{\begin{array}{l}
\dot{y}=\tilde{G}(y, t) \\
\dot{z}=2 \sqrt{1-z^{2}}
\end{array}, \quad y \in U, z(0)=0\right.
$$

is naturally defined on the torus $U \times[0,1] / \sim$ where $\sim$ is the equivalence relation

$$
(u, t) \sim(v, t)
$$

if and only if $u=v$ and $t=0$ or $\pi$.

Proof. The proof follows from the above discussion and the fact that the unique solution of the equation

$$
\dot{z}=2 \sqrt{1-z^{2}}, \quad z(0)=0
$$

is

$$
z(t)=\sin 2 t
$$

which is periodic with period $\pi$.

Example 5.1 Consider the trivial system

$$
\begin{aligned}
& \dot{x}_{1}=0 \\
& \dot{x}_{2}=0
\end{aligned}, \quad 0 \leq t \leq \pi
$$


defined on the disk $\{\|x\|<1\}$, and let $G$ be the orthogonal group $O(2, \mathbb{R})$. Then if

$$
G(t)=\left(\begin{array}{ll}
\cos t & \sin t \\
-\sin t & \cos t
\end{array}\right)
$$

(i.e. counterclockwise rotation through $t$ ), we have

$$
\begin{aligned}
\dot{y}(t) & =\dot{G}(t) G^{-1}(t) y(t)+G(t) \cdot\left(\begin{array}{l}
0 \\
0
\end{array}\right) \\
& =\left(\begin{array}{ll}
-\sin t & \cos t \\
-\cos t & -\sin t
\end{array}\right) \cdot\left(\begin{array}{ll}
\cos t & -\sin t \\
\sin t & \cos t
\end{array}\right) \cdot y(t) \\
& =\left(\begin{array}{ll}
0 & 1 \\
-1 & 0
\end{array}\right) \cdot y(t) .
\end{aligned}
$$

Hence the system

$$
\begin{aligned}
\dot{y}(t) & =\left(\begin{array}{ll}
0 & 1 \\
-1 & 0
\end{array}\right) \cdot y(t) \\
\dot{z} & =2 \sqrt{1-z^{2}}, \quad z(0)=0
\end{aligned}
$$

has trefoil knot solutions (see Fig 12).

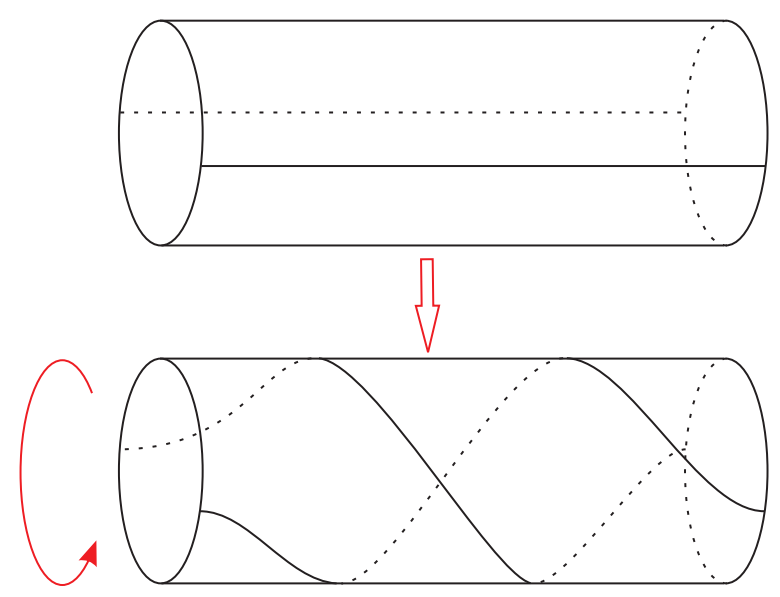

Figure 12: Forming a trefoil knot

This is, of course, a trivial example and to be useful we often require to operate in different regions of the state space with different 'local' transformation groups. To do this we introduce, as in the previous sections, $C^{\infty}$ functions defined on disjoint subsets of $\mathbb{R}^{n}$ as follows. Let $U_{i}, 1 \leq i \leq K$ (for some finite $K$ ) be bounded open subsets of $\mathbb{R}^{n}$ such that there exist disjoint open neighbourhoods $V_{i}$ of $U_{i}$ for which

$$
V_{i} \supseteq \overline{U_{i}} \text { and } V_{i} \cap V_{j}=\emptyset, \quad i \neq j, \quad 1 \leq i, j \leq K .
$$


Let $\varphi_{i}$ be a $C^{\infty}$ function such that

$$
\varphi_{i}(x)= \begin{cases}1 & , \text { if } x \in U_{i} \\ 0 & , \text { if } x \in \mathbb{R}^{n} \backslash V_{i} .\end{cases}
$$

Now let $G^{i}, 1 \leq i \leq K$ be $K$ (linear) transformation groups and let $t \rightarrow$ $G^{i}(t)$ be $K$ smooth functions with values in $G^{i}$. Then as in Equation 4 we consider the system

$$
\dot{x}=f(x, t)
$$

and the transformed system

$$
\dot{y}=\sum_{i=1}^{K} \varphi_{i}(y) \tilde{G}^{i}(y, t)
$$

where

$$
\tilde{G}^{i}(y, t)=\dot{G}^{i}(t)\left(G^{i}\right)^{-1}(t) y+G^{i}(t) f\left(\left(G^{i}\right)^{-1}(t) y, t\right) .
$$

Consider the effect of $G^{i}$ on $V_{i}$ at $t=\pi$. Define

$$
W_{i}=G^{i}(\pi) V_{i}
$$

and let

$$
X_{i j}=V_{i} \cap W_{j}, \quad 1 \leq i, j \leq K
$$

be the $K^{2}$ intersections of the sets $\left\{V_{i}\right\}$ and $\left\{W_{i}\right\}$. We assume that the functions $t \rightarrow G^{i}(t)$ are chosen so that the $X_{i j}$ are mutually disjoint. Let $\varphi_{i j}$ be the obvious restriction of $\varphi_{i}$ to $X_{i j}$ and consider the system

$$
\dot{y}=\sum_{i=1}^{K} \varphi_{i j}(y) \tilde{G}^{i}(y, t) .
$$

Theorem 5.2 Using the above notation, if the function

$$
\sum_{i=1}^{K} \varphi_{i j}(y) \tilde{G}^{i}(y, t)
$$

is periodic with period $\pi$, then the system

$$
\begin{aligned}
& \dot{y}=\sum_{i=1}^{K} \varphi_{i j}(y) \tilde{G}^{i}(y, t), \quad y \in U, 0 \leq t \leq \pi \\
& \dot{z}=2 \sqrt{1-z^{2}}, \quad z(0)=0
\end{aligned}
$$

(where $U$ is a ball containing all sets $V_{i}$ ), is naturally defined on the torus $U \times[0,1] / \sim$ where $\sim$ is as in Theorem [5.1. 
Example 5.2 We will use this method to generate systems with arbitrarily knotted chaos. Consider first a system with unknotted chaos. Let $U_{1}, U_{2}$ be the sets

$$
\begin{aligned}
& U_{1}=\left\{\left(x_{1}, x_{2}\right): 0<x_{1}<1,0<x_{2}<\frac{1}{3}\right\} \\
& U_{2}=\left\{\left(x_{1}, x_{2}\right): 0<x_{1}<1, \frac{2}{3}<x_{2}<1\right\}
\end{aligned}
$$

and $W_{1}, W_{2}$ the sets

$$
\begin{aligned}
& W_{1}=\left\{\left(x_{1}, x_{2}\right): 0<x_{1}<\frac{1}{3}, 0<x_{2}<1\right\} \\
& W_{2}=\left\{\left(x_{1}, x_{2}\right): \frac{2}{3}<x_{1}<1,0<x_{2}<1\right\} .
\end{aligned}
$$

The transformation groups $G_{1}, G_{2}, G_{3}$ correspond to: the 'stretch and squeeze'

$$
G_{1}(t):\left(x_{1}, x_{2}\right) \rightarrow\left(\frac{x_{1}}{t}, t x_{2}\right),
$$

rotation

$$
G_{2}(t):\left(x_{1}, x_{2}\right) \rightarrow\left(t\left(x_{2}-2.5\right),-t\left(x_{1}-\frac{1}{6}\right)\right)
$$

and translation

$$
G_{3}(t):\left(x_{1}, x_{2}\right) \rightarrow\left(x_{1}+t, x_{2}-t\right) .
$$

Finally we define

$$
X_{i j}=V_{i} \cap W_{j}, \quad 1 \leq i, j \leq 2,
$$

and we have the system

$$
\begin{aligned}
& \dot{y}=\sum_{i, j=1}^{2} \varphi_{i j}(y) \tilde{G}^{i}(y, t) \\
& \dot{z}=2 \sqrt{1-z^{2}}
\end{aligned}
$$

where $\varphi_{i j}(y)$ is a $C^{\infty}$ function corresponding to $X_{i j}$,

$$
G^{1}=G_{1}, \quad G^{2}=G_{3} \circ G_{2} \circ G_{1}
$$

and $\tilde{G}^{i}$ is obtained from $G^{i}$ as in Equation [5, This system has chaotic orbits as shown in Fig 13, Note that if $G_{i}(t), \quad 1 \leq i \leq 3$ are properly chosen, the system has no homoclinic orbits. (This simply implements the 'Smale horseshoe' map.)

Now consider a system of the form

$$
\begin{aligned}
\dot{x} & =f(x, t) \\
\dot{z} & =2 \sqrt{1-z^{2}}
\end{aligned}
$$




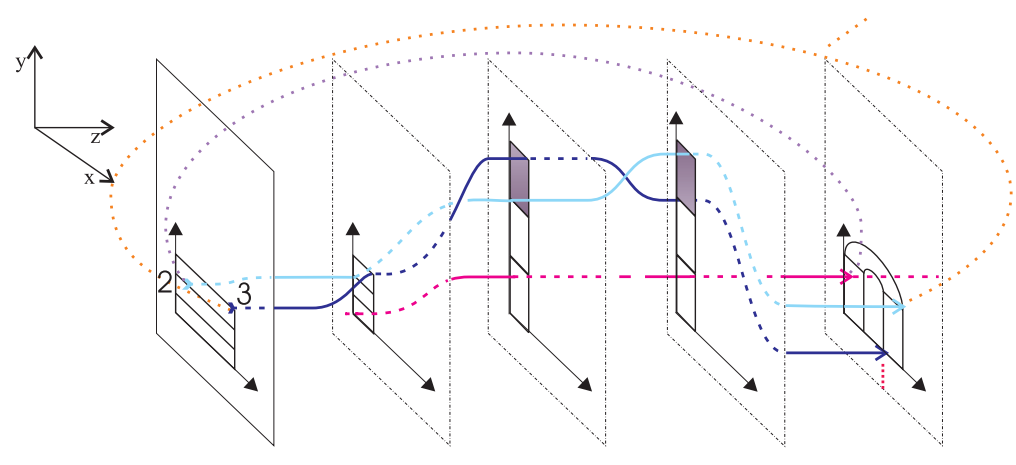

Figure 13: Creating a chaotic system from the Smale horseshoe

defined for $x \in U$, where $U$ is some bounded open set in $\mathbb{R}^{n}$. Let $\psi$ : $[0, \pi] \rightarrow \mathbb{R}^{n}$ be any $C^{\infty}$ function (which represents a strand of a braid) and let $\varphi: \mathbb{R}^{n} \rightarrow \mathbb{R}$ be a $C^{\infty}$ function which is 1 on $U$ and 0 outside some neighbourhood of $U$. Then, if we put

$$
y=x+\psi
$$

the system

$$
\begin{aligned}
\dot{y} & =\dot{x}+\dot{\psi}=(f(x, t)+\dot{\psi}) \varphi(x-\psi(t)) \\
& =(f(y-\psi, t)+\dot{\psi}) \varphi(y)
\end{aligned}
$$

has trajectories like those of Equation 17 in $U$, but 'bent' by $\psi$. (See Fig 14)

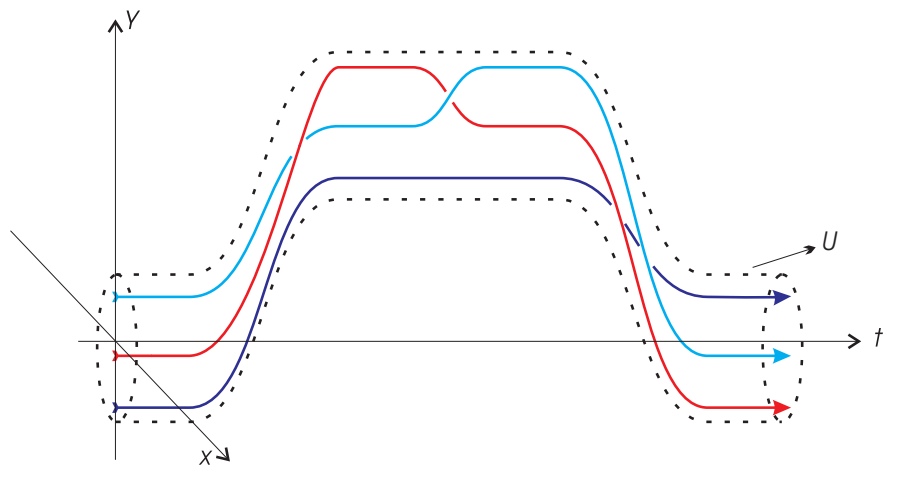

Figure 14: A twisted braid

More generally, if $U_{i}, 1 \leq i \leq K$, are several open (disjoint) sets in $\mathbb{R}^{n}$, 
and $\varphi_{i}, \psi_{i}$ are associated functions as above, then the system

$$
\begin{aligned}
& \dot{y}=\sum_{i}\left(f\left(y-\psi_{i}(t), t\right)+\dot{\psi}_{i}(t)\right) \varphi_{i}\left(x-\psi_{i}(t)\right) \\
& \dot{z}=2 \sqrt{1-z^{2}}
\end{aligned}
$$

will have trajectories similar to a given system in the regions $U_{i}$, but 'bent' by the functions $\psi_{i}$. Clearly, by appropriate choice of $\psi_{i}$ and $\varphi_{i}$ we can obtain a system with arbitrarily knotted chaos, which contains no homoclinic orbits. Fig 15]shows a braid representation of a trefoil knot which contains a chaotic system, Smale horseshoe, inside.

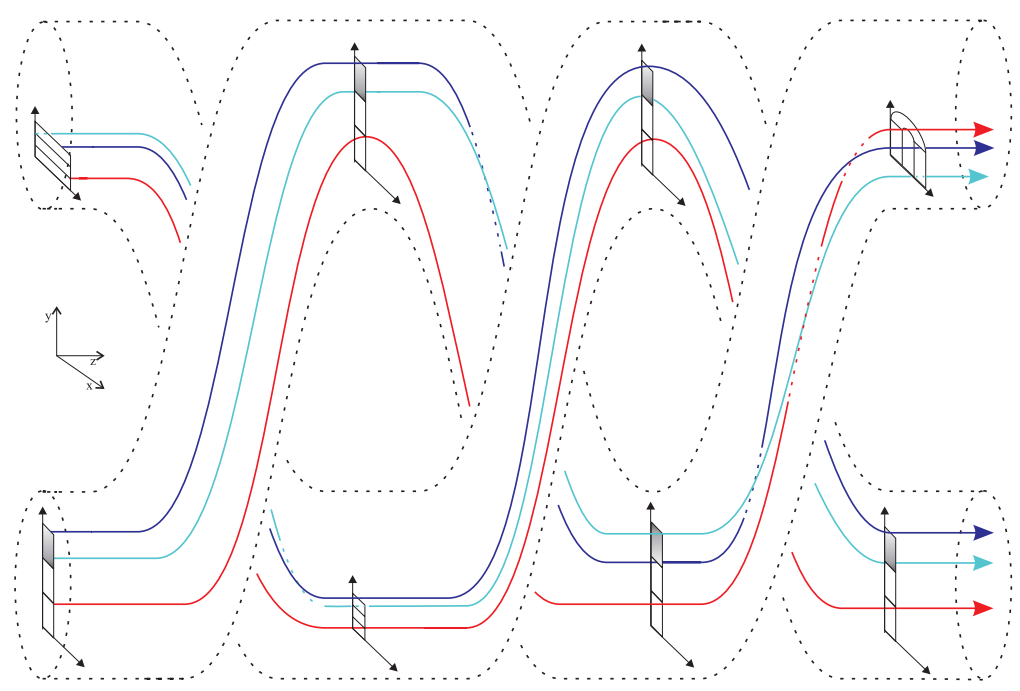

Figure 15: Knotted chaos

\section{Conclusions}

In this paper we have shown how to generate three-dimensional systems containing arbitrarily knotted chaos by using the theory of transformation groups and $C^{\infty}$ functions. By 'twisting' a simple existing dynamical system by local transformation groups and making the resulting system periodic, virtually any dynamical behaviour can be obtained. In the second part of the paper we shall consider more general three-manifolds and dynamical systems defined on them by using the theory of Heegaard splittings. Every three-manifold has a Heegaard splitting which represents it in the form of two three manifolds with genus $p$ surfaces glued together along a framed knot. 


\section{References}

[1] Artin, E. [1925] "Zur Isotopie zwerdimensionaler Flachen im $R_{4}$ ", Hambrug Abh., 4, 174-177.

[2] Banks, S. P. and Diaz, D. [2004] "Almost radially-invariant systems containing arbitrary knots and links", Int. J. of Bifurcation and Chaos, 14, $1267-1276$.

[3] Banks, S. P. and Song, Y. [2006] "Elliptic and automorphic dynamical systems on surfaces", Int. J. of Bifurcation and Chaos, in press.

[4] Birman, J. and Williams, R. [1983] "Knotted periodic orbits in dynamical systems - I: Lorenz' Equations", Topology, 22, 47-82.

[5] Ghrist, R. W., Holmes, P. J. and Sullivan, M. [1997] "Knots and links in three-dimensional flows", New york: Spring-Verlag, LNM, 1654.

[6] Kauffman, L. [1991] "Knots and physics", Singapore: World Sci. Pub. 